\title{
LA ACTUACIÓN DEL ESTADO EN MATERIA TURÍSTICA DURANTE LA DICTADURA DE PRIMO DE RIVERA
}

CARMELO PELLEJERO MARTÍNEZ

Universidad de Málaga

Durante la primera mitad del siglo $\mathrm{xx}$, es decir, antes de que el viajar a nuestro país se convirtiera en un fenómeno de masas, el Estado español no fue indiferente con respecto al turismo. Es cierto que su desarrollo no fue nunca algo prioritario para las autoridades, pero por la importancia social, cultural, política y, sobre todo, económica del turismo, todas las administraciones públicas que se sucedieron a lo largo del período se preocuparon por fomentarlo y controlarlo. Aunque, naturalmente, no siempre con los mismos medios, ni con los mismos instrumentos, ni, por supuesto, con el mismo éxito. La etapa más interesante de cara al desarrollo del turismo fue la de la Dictadura de Primo de Rivera. Hasta que se produjo el levantamiento militar, la actuación de la Administración pública en materia turística había sido bastante limitada. Con escasos recursos humanos y económicos, ésta se había centrado especialmente en la promoción de España en el exterior y en acrecentar, conservar y difundir nuestro patrimonio artístico y cultural. Sin embargo, esta política experimentó un notable cambio desde mediados de la década de los años veinte. Por primera vez en la historia los responsables turísticos de la Administración actuaron teniendo en cuenta los variados aspectos que se relacionan con el turismo. Se consideró prioritario, especialmente ante la prevista celebración en 1929 de la Exposición Iberoamericana de Sevilla y de la Exposición Internacional de Barcelona, ampliar y mejorar la infraestrućtura hotelera, crear un servicio que, tanto en el interior como en el exterior del país, informara verazmente sobre nuestros atractivos turísticos, incrementar nuestros sistemas de trans- 
porte y la calidad de los mismos, y fomentar las actividades artísticas, deportivas, festivas y congresuales como reclamos turísticos.

\section{ORGANIZACIÓN ADMINISTRATIVA Y ACTIVIDADES}

Cuando el 13 de septiembre de 1923 Miguel Primo de Rivera tomó el poder en España, la organización administrativa del turismo correspondía a la Comisaría Regia del Turismo y Cultura Artística. Este organismo había sido creado en 1911 con la misión de procurar el desarrollo del turismo y la divulgación de la cultura artística popular. En sus doce años de existencia, buena parte de ellos marcados por la I Guerra Mundial y su consiguiente postguerra, se había preocupado fundamentalmente de promocionar España en el extranjero y de incrementar y cuidar el patrimonio cultural español. La Comisaría, o mejor dicho su Comisario Regio, Benigno Vega Inclán y Flaquer, que siempre desempeñó su cargo de manera gratuita, había conseguido, a pesar de las escasas dotaciones presupuestarias incluidas en las correspondientes al Ministerio de Instrucción Pública y Bellas Artes, crear museos, construir jardines, organizar y participar en exposiciones de arte, conservar monumentos, fundar bibliotecas y archivos, y editar un buen número de publicaciones en las que los turistas podían encontrar, desde posibles itinerarios para conocer nuestra geografía hasta estudios pormenorizados de la riqueza artística de diversas ciudades monumentales españolas.

En un primer momento, las autoridades primorriveristas no prestaron demasiado atención al turismo y decidieron que la Comisaría Regia siguiera al frente de la organización administrativa del mismo. Y, curiosamente, en las mismas condiciones. Es decir, continuaría siendo un órgano escasamente dotado y excesivamente personalizado. No obstante, con el paso del tiempo la Administración fue convenciéndose de la necesidad de crear un nuevo organismo estatal que, dotado con mayores recursos económicos y humanos de los que había contado la Comisaría, fuera capaz de atender eficazmente a los variadísimos aspectos que se relacionan con el turismo. Y en abril de 1928 creó el Patronato Nacional del Turismo (PNT).

A diferencia de lo ocurrido con la Comisaría Regia, el nuevo organismo contó con un presupuesto propio. Se estipuló que su financiación correría a cargo de los recursos que se obtuvieran con la creación de un seguro obligatorio de las personas que viajaran por ferrocarril y del ganado vivo que se transportara en él. Además, se indicó que hasta que dicho seguro 
entrara en funcionamiento, cosa que no ocurriría hasta octubre de 1928, el Tesoro anticiparía al PNT, con carácter reintegrable, hasta 250.000 pesetas, que iría disponiendo por sucesivos libramientos en función de sus necesidades. Asimismo, éste quedó facultado en noviembre de 1928 para concertar con la banca nacional un empréstito de 25 millones de pesetas como fondo inicial de funcionamiento.

Desde la Administración pública se consideró prioritario de cara al desarrollo del turismo ampliar y mejorar la infraestructura hotelera española. De la labor desarrollada en este sentido hay que resaltar la creación de: 1) Servicio de Crédito Hotelero, con la finalidad de estimular y auxiliar la construcción de hoteles por parte de la iniciativa privada; 2) Título de Establecimiento Recomendado, como estímulo para el mejoramiento de las industrias relacionadas con el turismo, y especialmente la hotelera, y como garantía para el viajero; 3) Cámara Oficial Hostelera, con la misión de perseguir la clandestinidad, evitar los abusos y organizar la industria del hospedaje; 4) Guía Oficial, con el objetivo de proporcionar a los viajeros una relación de alojamientos y de sus precios; 5) Libro de Reclamaciones, para que los viajeros pudieran anotar en él cuantas anomalías observaran durante su estancia en los establecimientos hoteleros; y 6) Red de Paradores y Albergues de Carretera, una oferta hotelera pública caracterizada por precios ajustados, servicios de calidad e instalaciones confortables que se ubicarían en áreas turísticamente atractivas pero con una escasa o inexistente infraestructura hotelera privada.

A mediados de la década de los años veinte España continuaba siendo un país poco conocido en el exterior. $\mathrm{Y}$ esto era, lógicamente, un severo lastre para el desarrollo del turismo. Era urgente aliviar dicho desconocimiento. Para ello el PNT abrió al público siete agencias informativas en el extranjero, concretamente en París, Londres, Múnich, Roma, Gibraltar, Nueva York y Buenos Aires, y alrededor de medio centenar en territorio español. Además, tanto la Comisaría Regia como el PNT hicieron un valioso esfuerzo editorial de cara a la divulgación turística de nuestro país. Una serie de excelentes publicaciones, profusamente ilustradas y redactadas en varios idiomas, facilitaron la visita a España, tanto en el sentido de proporcionar datos prácticos de carácter general, como en el de ofrecer al turista en cualquier punto de España los elementos necesarios para su guía, tanto en las necesidades materiales y prácticas de su viaje, como en los datos históricos, artísticos y descriptivos.

Practicar turismo implica viajar. Por lo tanto, el desarrollo turístico de un país está muy condicionado por los medios de transporte de que 
dispone. En la medida en que éstos sean variados, rápidos, cómodos, seguros y económicos, se facilitará el movimiento de viajeros de un lugar a otro. Pues bien, sabemos que durante la Dictadura de Primo de Rivera se prestó una atención especial al incremento y mejora de nuestros sistemas de transporte. Se mejoró la red ferroviaria y se renovó y amplió el material móvil utilizado en la misma; se creó el Circuito Nacional de Firmes Especiales, que consiguió en apenas tres años y medio el arreglo de 2.800 kilómetros de carreteras; y se avanzó significativamente en el todavía incipiente transporte aéreo. Lógicamente, todo ello fue muy positivo de cara al turismo. Pero también lo fue el hecho de que el PNT consiguiera que algunas compañías de transportes terrestres y marítimos contribuyeran, mediante la concesión de determinadas facilidades a sus clientes, a incentivar el movimiento de viajeros, tanto hacia España como en el interior de la nación.

Las autoridades primorriveristas, en su afán de promover una gran corriente del turismo internacional hacia España y de fomentar el turismo interior, utilizaron como principales reclamos turísticos, al margen de las exposiciones de Sevilla y Barcelona, las actividades artísticas y deportivas, las fiestas populares y la celebración de congresos. En otoño de 1928 el PNT organizó una importante exposición de fotografías de carácter turístico en el Palacio de Bibliotecas y Museos de Madrid. Poco después, en enero de 1929 montó otra exposición similar en la Sociedad Española de Amigos del Arte de Madrid. A lo largo de dicho año organizó, asimismo, Exposiciones Regionales de Arte en Valencia, Toledo, Santillana del Mar y Granada, y una serie de excursiones para que un buen número de periodistas extranjeros conocieran Madrid, Barcelona, Sevilla y Toledo. Por lo que respecta al deporte, el PNT dedicó una especial atención al golf, las regatas, la hípica y el motorismo. También promocionó algunas fiestas populares, como las Fallas de Valencia y los festivales de Castellón, Alicante y San Sebastián. Y, por último, intervino directa o indirectamente en la organización de festejos con motivo de la celebración de diversos congresos internacionales, como, por ejemplo, el IX Congreso Internacional de Agencias de Viajes y el Primer Viaje y Congreso de Estudios Médicos, celebrados en octubre de 1928 y junio de 1929, respectivamente.

\section{ACTUACIÓN ECONÓMICA}

Hay varios aspectos que merece la pena destacar a la hora de analizar la aportación económica de la Dictadura de Primo de Rivera en pro del 
turismo. En primer lugar, hay que señalar que el gasto estatal en materia turística fue muy criticado por su escasez durante todo el período, pero especialmente hasta la creación del PNT. El Comisario Regio acusó reiteradamente a las autoridades gubernativas de cierta racanería a la hora de apoyar el desarrollo turístico. El máximo responsable de la Comisaría Regia expresó frecuentemente su malestar por los insuficientes recursos económicos, y también humanos, con los que generalmente tuvo que hacer frente a la tarea que se le había encomendado. En segundo lugar, habría que destacar que las asignaciones económicas que dispusieron la Comisaría Regia y el PNT fueron crecientes, al menos en términos absolutos, con el paso del tiempo. Desde el 1 de abril de 1924 al $31^{\circ}$ de diciembre de 1927, es decir, la etapa en la que la Comisaría Regia continuó al frente del turismo español, el Estado le asignó a través del Ministerio de Instrucción Pública y Bellas Artes una media de 11.200 pesetas al mes. Esta aportación, duramente criticada por Vega Inclán, fue en términos absolutos superior a lo gastado en los años anteriores al golpe militar. Por ejemplo, entre el 1 de abril de 1919 y el 31 de marzo de 1924, el gasto medio mensual había sido de 8.166 pesetas.

Como se señaló anteriormente, a finales del mes de abril de 1928 la Comisaría Regia fue sustituida por el PNT, un órgano ahora dependiente del Ministerio de la Presidencia y al que se le anticiparon 250.000 pesetas para lo que quedaba de año, es decir, aproximadamente la mitad de todo el dinero que había recibido la Comisaría durante los ejercicios de 1924, 1925, 1926 y 1927. Además, en el año 1929 se introdujo una importante novedad. El máximo organismo público en materia turística contó, por vez primera en la historia, con un presupuesto propio. ¿En qué emplearon sus recursos la Comisaría Regia y el PNT? Por lo que respecta al primer organismo, desgraciadamente no contamos con datos estadísticos fiables. $\mathrm{Si}$ nos atenemos a las opiniones de su máximo responsable, el dinero se empleó, fundamentalmente, en labores de propaganda y de conservación del patrimonio artístico. En cambio, gracias a la Memoria publicada en 1930 por el PNT, sí disponemos de información cuantitativa para analizar con detenimiento la actividad económica desarrollada por dicho organismo en los años 1928 y 1929.

El PNT comenzó los trabajos de organización en los primeros días del mes de junio de 1928. Y lo hizo sin recursos. Éstos no llegarian hasta el día 18 de agosto de dicho año cuando recibió del Tesoro, con cargo a un anticipo de 250.000 pesetas, las primeras 150.000 . Las restantes 100.000 pesetas no las tendría en su poder hasta el día 19 de octubre. 
La liquidación de operaciones realizada en 31 de diciembre de 1928 arrojó una cifra total de gastos de 300.269 pesetas. Estos gastos fueron ocasionados por la puesta en marcha de las oficinas centrales y regionales y de alguna de las agencias informativas ubicadas en el extranjero ( 72 por 100 ), la publicación y adquisición de folletos, guías, planos de carreteras $(26,2$ por 100$)$ y la concesión de subvenciones para el IX Congreso Internacional de Agencias de Viajes, la Regata Internacional Nueva York-Santander y la instalación del Pabellón del Turismo en la Exposición Iberoamericana de Sevilla (1,8 por 100). Para el ejercicio económico de 1929 el PNT presentó un Presupuesto Ordinario, con cargo al cual se dotaron aquellas atenciones de sostenimiento y acción constante y regular; un Presupuesto Extraordinario de Gastos, en el que fueron comprendidas las operaciones derivadas de una intensa actuación extraordinaria y eventual; y, por último, un apartado denominado Inversión de Capital, en el que se incluyeron los conceptos que, no suponiendo gastos, constituían una aplicación decidida de capital, en la Caja u operaciones de préstamos por el Crédito Hotelero ( 10.000 pesetas), y el sobrante de fondos, representado en un Fondo de Reserva (3.970.500 pesetas). Los presupuestos citados respondían a un criterio de nivelación de los distintos gastos con los recursos calculados. Así, el Presupuesto Ordinario de gastos quedó limitado a 8 millones de pesetas y el Extraordinario y la Inversión de Capital suponían en conjunto 23.500 .000 pesetas, obtenida del recurso extraordinario originado por el empréstito de 25 millones de pesetas emitido, tal y como se señaló en páginas precedentes, como fondo inicial de funcionamiento por el PNT.

Los ingresos presupuestados para el ejercicio de 1929 ascendieron a 31.500 .000 pesetas. Sin embargo, los realmente obtenidos fueron 27.180.573 pesetas. Este desfase fue consecuencia, sobre todo, de que el rendimiento calculado en beneficio del PNT por el seguro obligatorio sobre el transporte de viajeros (3.361.017 pesetas) fue bastante inferior al presupuestado (7.500.000 pesetas). Esto obedeció a que al finalizar el año la Comisaría del Seguro Obligatorio todavía no había entregado al PNT el resultado de la liquidación de los meses de noviembre y diciembre, en el que se comprendía la recepción del 25 por 100 de la recaudación total del seguro que la Comisaría hacía, y la parte que pudiera corresponder al PNT en la recaudación del cuarto trimestre de 1929. Por otro lado, también se ingresó menos de lo previsto por la venta de publicaciones. Concretamente el 20 por 100 de lo presupuestado. La justificación hay que buscarla en la demora experimentada en la realización del plan de 
publicaciones y, consiguientemente, en la organización del servicio de distribución para las ventas. Por último, sólo los ingresos obtenidos por razón de intereses a favor del PNT en la colocación de sus fondos en cuentas corrientes abiertas en bancos particulares (23.799.539 pesetas) fueron prácticamente idénticos a los previamente calculados (23.850.000 pesetas).

El cálculo de los créditos ascendió a 8 millones de pesetas. El presupuesto se articuló en 14 capítulos: $1 .^{\circ}$ Anualidad para pago de intereses y amortización primera del empréstito emitido (1.750.000 ptas.); $2 .^{\circ}$ y 3. Personal y material de las oficinas centrales del PNT y alquileres de los locales ocupados por las mismas $(324.000,40.000$ y 20.000 ptas., respectivamente); $4 .^{\circ}$ Mantenimiento de las cinco subdelegaciones regionales del PNT (306.000 ptas.); $5 .^{\circ}$ Sostenimiento de las Juntas provinciales y locales (250.000 ptas.); $6 .^{\circ}$ Personal, material y alquileres de las agencias organizadas en París, Londres, Nueva York, Berlín y Buenos Aires (800.000 ptas.); $7 .^{\circ}$ Necesidades derivadas de los viajes de gestión por todo el personal del PNT y para asistencias al Consejo General del Turismo (100.000 ptas.);.$^{\circ}$ Propaganda ordinaria mediante publicaciones, prensa, etc., en España y en el extranjero (1.000.000 de ptas.); 9. Entretenimiento de material del Teatro Real, conservación y administración del Parador de Gredos y de la Casa de los Tiros de Granada, y subvención fija para el Patronato especial del Museo Romántico, Casa del Greco en Toledo y Casa de Cervantes en Valladolid (120.000 ptas.); $10 .^{\circ}$ Posible déficit en la explotación y administración de 20 albergues de carreteras y de los paradores y hosterías que pudieran abrirse al público (140.000 ptas.); $11 .^{\circ}$ Organización y sostenimiento de escuelas hoteleras y de guías (100.000 ptas.); 12. ${ }^{\circ}$ Crédito necesario para la realización de todas aquellas garantías de interés que, dentro del máximo del 5 por 100, había concedidas, o pudieran concederse, a particulares o entidades que invirtieron capital en la construcción de nuevos hoteles, establecimiento de autobuses de turismo, construcción de campos de golf y otras obras de interés turístico $(1.200 .000$ ptas.); $13 .^{\circ}$ Crédito para toda clase de subvenciones a congresos, exposiciones y otros motivos de atracción turística, e incluso para la adquisición de edificios de carácter histórico o artístico que debieran pasar a la propiedad del Estado (1.500.000 ptas.); $14 .^{\circ}$ Imprevistos (350.000 ptas.).

De los 8 millones de pesetas presupuestados, se liquidaron realmente 5.215.355 pesetas. Los capítulos donde los gastos reales estuvieron más alejados de los previstos fueron el $1 .^{\circ}$, el $100^{\circ}$, el $11 .^{\circ}$ y el $12 .^{\circ}$ En el primer caso, por intereses de bonos se pagaron 932.916 pesetas, en lugar de las 1.250 .000 presupuestadas, debido a no haberse realizado en la fecha 
de liquidación el pago del cupón del mes de enero de 1930. Además, por el concepto de amortización de bonos, las 500.000 pesetas presupuestadas para el pago de la primera amortización del empréstito, quedaron como remanente por no corresponder dicha amortización hasta el año 1930. En cuanto al capítulo $10 .^{\circ}$, sólo se liquidó el 35,7 por 100 de la cantidad presupuestada por algunos retrasos registrados en la construcción de los establecimientos públicos previstos. En cuanto a los capítulos $11 .^{\circ}$ y $12 .^{\circ}$, como no fueron consumidos por pago alguno, a finales de 1929 fueron anulados los créditos.

Con cargo al producto de la negociación del empréstito, se reunieron en un Presupuesto Extraordinario de gastos todas aquellas dotaciones que respondían a conceptos derivados de actuaciones especiales y eventuales del PNT. El importe total de este presupuesto ascendió a 9.529 .500 pesetas, según el detalle siguiente: $1 .^{\circ}$ Créditos necesarios para la devolución al Tesoro Público de su anticipo reintegrable de 250.000 pesetas, hecho al PNT en el año anterior, y para liquidar el déficit del ejercicio de 1928 (250.000 y 100.000 ptas., respectivamente); $2{ }^{\circ}$ Gastos de emisión del empréstito (222.500 ptas.); 3. ${ }^{\circ}$ Créditos necesarios para la instalación definitiva de las oficinas centrales, de las cinco subdelegaciones regionales y de las cinco agencias del PNT en el extranjero (1.075.000 ptas.); $4 .^{\circ}$ Reformas urgentes en el Parador de Gredos y en la Casa de los Tiros de Granada (32.000 ptas.); 5. ${ }^{\circ}$ Ayuda a la construcción del Hotel Atlántico de Cádiz, albergues de carretera y paradores $\left(4.200 .000\right.$ ptas.); 6. ${ }^{\circ}$ Organización e instalación de exposiciones regionales de arte moderno y de industrias artísticas ( 350.000 ptas.); $70^{\circ}$ Instalación y presentación adecuada del Tesoro Artístico Nacional (300.000 ptas.); $8^{\circ}$ Instalación y entretenimiento del pabellón del PNT en la Exposición Iberoamericana de Sevilla (300.000 ptas.); $9^{\circ}$ Propaganda de carácter turístico en el extranjero (1.500.000 ptas.); $10{ }^{\circ}$ Construcción e instalación de 22 oficinas de información al público en el interior del país (1.000.000 de ptas.); $11 .^{\circ}$ Temporada de Ópera en Madrid (200.000 ptas.).

Del Presupuesto Extraordinario de gastos se pagaron 5.332 .516 pesetas. Independientemente de los conceptos de cancelación del déficit del PNT y gastos de emisión del empréstito, más los de instalación de las oficinas centrales, regionales y del extranjero, aparecen como gastos principales los realizados con cargo a los siguientes conceptos: 1) por lo que respecta a la ayuda al Hotel Atlántico de Cádiz, hay que señalar que la cantidad pagada coincide con la presupuestada, 1.600 .000 pesetas; 2) sin embargo, de los créditos presupuestados para construcción e instalación de albergues, 
paradores y hosterías, 2.600 .000 pesetas, sólo se pagaron 263.042 pesetas; 3 ) en el capítulo $6 .^{\circ}$ se autorizó un crédito de 350.000 pesetas contra el que se realizaron pagos sólo por 162.000 pesetas; 4) en la instalación y mantenimiento del pabellón del PNT en la exposición de Sevilla se gastaron 220.152 pesetas, un 73,4 por 100 de lo previsto; 5) del 1.500 .000 pesetas presupuestado para tareas de propaganda en el extranjero, se gastó en realidad el 94,3 por 100 .

\section{A MODO DE CONCLUSIÓN}

¿Cuáles fueron las principales aportaciones en materia turística durante la dictadura de Primo de Rivera? En primer lugar, la administración pública primorriverista fue la primera que entendió que para atraer un número creciente de forasteros al país, o con otras palabras, que para incrementar las divisas que tanto necesitaba nuestra economía, era preciso actuar sobre todos los aspectos que, en mayor o menor medida, inciden en el turismo. No bastaba con promocionar el país. Tampoco era suficiente el arte como producto turístico. Era preciso, además, ofertar más productos e incrementar y mejorar los transportes y los establecimientos hoteleros. $\mathrm{Y}$ en segundo lugar, hay que destacar el intento de las autoridades gubernativas de dotar de más medios humanos y económicos al máximo órgano responsable del turismo español. Lo hizo dotando al PNT de un presupuesto propio, algo con lo que no contaron sus organismos predecesores. Pero la experiencia fue muy corta. Tras la dimisión de Primo de Rivera se inició una etapa muy convulsa para el PNT. Experimentó su primera reforma en julio de 1930 y durante la República fue liquidado en abril de 1931 y restablecido en diciembre de dicho año. A partir de ese momento el presupuesto del PNT pasó a depender del de Presidencia del Gobierno. La extinción definitiva del PNT llegaría durante la Guerra Civil.

\section{BIBLIOGRAFÍA}

Comisaría Regia del Turismo (1917): Obra encomendada a la Comisaria Regia del Turismo y recursos para realizarla, Madrid.

- (1925): Solicitudes e Informes de ia Comisaria Regia del Turismo, Madrid.

Fernández Álvarez, J. (1974): Curso de Derecho Administrativo Turístico, Madrid, Editora Nacional.

FERNÁNDEZ FuSTER, L. (1991): Historia general del turismo de masas, Madrid, Alianza. 
LaVAuR, L. (1980): «Turismo de entreguerras, 1919-1939», Estudios Turísticos, 67 y 68, pp. 11-112 y 13-129.

Patronato Nacional del Turismo (1930): Memoria de los trabajos realizados por el Patronato Nacional del Turismo desde julio de 1928 a 31 de diciembre de 1929, Madrid, Talleres Voluntad.

Pellejero Martínez, C. (dir.) (1999): Historia de la economía del turismo en España, Madrid, Civitas.

VEGA INCLAN, Marqués de la (1927): Turismo en España, Madrid. 